\title{
COOPERATIVE AD-HOC NETWORKS FOR ENERGY EFFICIENT IMPROVE CONNECTIVITY
}

\author{
Mohua Biswas ${ }^{1}$, M.S.Mathpati ${ }^{2}$, Papiya Biswas ${ }^{3}$ \\ ${ }^{I}$ Assistant Professor, Electronics \& Telecommunication Department, SVERI's C.O.E.Pandharpur, Maharashtra, India \\ ${ }^{2}$ Assistant Professor, Electronics \& Telecommunication Department, SVERI's C.O.E.Pandharpur, Maharashtra, India \\ ${ }^{3}$ Assistant Professor, Electronics \& Telecommunication Department, SVERI's C.O.E.Pandharpur, Maharashtra, India
}

\begin{abstract}
Mobile ad-hoc network (MANET) has been developed to meet the increase in demand for high-speed wireless network. Wireless adhoc networks are multi-hop frameworks comprising of connections among wireless nodes in the absence of any infrastructure. Thus they normally consist of unpredicted network topologies. In MANETs nodes have the liberty to move freely in various directions. Due to limited battery power, nodes require energy efficient routing protocols to gear up their network performance. Cooperative communication plays an important role in wireless networks. This is evident from the fact that most of the present cooperative communication works are aimed at link-level physical layer issues. As a consequence, the influence of cooperative communication on network-level upper layer issues, such as topology control, routing and network capacity is unnoticed. Cooperative communication is a technology by the help of which multiple nodes transmit similar data at the same time. This increases the transmission coverage and also saves power. Earlier research work on topology control dealt with cooperative communication in the form of energy saving only and did not consider for extending the coverage. It has been noticed that cooperative communication can link disconnected networks. To overcome this problem, we have studied energy efficient topology control with cooperative communication. In this paper, it has been proposed for selection of optimum relay nodes so as to decrease the overall power consumption in the cooperative ad-hoc network.
\end{abstract}

Keywords: Algorithm, Cooperative communication, Efficient Energy, Mobile Ad-hoc network, Energy efficiency, Topology. $* * *$

\section{INTRODUCTION}

The Wireless ad-hoc network has different civilian and defense applications and as such it has gained considerable attention in modern days. The main consideration in designing such a network is to reduce power consumption because of the fact that wireless nodes are normally charged by batteries only. Topology control is one of the major energy conserving technique which has been mainly considered and used in adhoc wireless network. Topology control technique permits each and every wireless node to select certain subset of neighbors and prolong network connectivity by consuming the minimum transmission power. Thus with the help and support of this technique, each node becomes competent to maintain its connectivity with various other nodes by one-hop or multihop even without utilizing the optimum transmission power. Thus by conserving energy, topology control helps in decreasing interference between wireless connections by reducing the number of links. Co-operative communication (CC) allows single antenna device to receive the benefit of the multiple-input and multiple- output (MIMO) systems. By examining in detail the transmission capacity of the wireless medium, cooperative communication allows nodes that have obtained transmitted signal to assist collectively in transferring information to other nodes.

\section{RELATED WORKS}

Topology control is an approach used in distributed computing to change the critical network (modeled as a graph) in order to reduce the cost of distributed algorithms if run through the new resulting graphs. It is a primary approach in distributed algorithm. For example, a (minimum) spanning tree is treated as a backbone to reduce the cost of transmission from source $S$ (x) to destination $\mathrm{D}(\mathrm{y})$, where $\mathrm{x}$ and $\mathrm{y}$ are considered in the graph as the number of lines and vertices respectively.

The term "topology control" is used frequently by the wireless ad-hoc and sensor networks research association. The main objectives of topology control, in this concern, are to conserve energy, reduce the obstruction between nodes and increase the lifespan of the network. In paper (1) and (5) under reference, the authors introduce energy-efficient topology control problem in cooperative ad-hoc networks and propose two topology control algorithms to build cooperative energy spanners wherein the energy efficiency of individual path is guaranteed. They suggested algorithms to carry out the 
performance in distributed and localized fashion by maintaining the globally efficient path. Recent study on the subject reveals significant performance gain in various energy efficient cooperative wireless networks (7) and (8), cooperative multicast scheduling scheme under reference (6), connectivity/coverage improvement [3], and relay selection for throughput maximization or energy conservation [4], [9] \& [12]. In paper [2], author proposes a Capacity-Optimized Cooperative (COCO) topology control program by utilizing both upper layer network capacity and physical layer cooperative communication so as to improve the network capacity in MANETs. In paper [13], author proposes a routing algorithm, the maximum energy level maximum ad-hoc distance vector based on alternate maximum energy remaining routes in each node so as to increase the lifespan of the network.

\section{COOPERATIVE COMMUNICATION}

Cooperative communication is defined as a process by which users contribute and combine resources relating to transmission of data to gear up their performances with the help of each other. This technique has the advantage to increase the transmission coverage of a node in mobile ad-hoc network on account of diverse channel quality, limited energy and limited bandwidth wireless environment. Due to cooperation in communication, users having weak network connection can utilize quality channels of their partners so as to achieve the desired quality of service. Wireless communication is a technique in a wireless ad-hoc network wherein users can improve their service quality through cooperative communication system. For example, in figure 1, node $\mathrm{A}$ is not able to communicate with node $\mathrm{B}$, since $\mathrm{B}$ is out of its ultimate transmission range of A. On the other hand, A can relay a cooperation request message and data to neighboring connected nodes $\mathrm{H}$ as relay node and then the three nodes all together pass on the data to B. As such, B can receive it as a result of extended transmission range of nodes $\mathrm{A}, \mathrm{H}$, and $\mathrm{H}$.

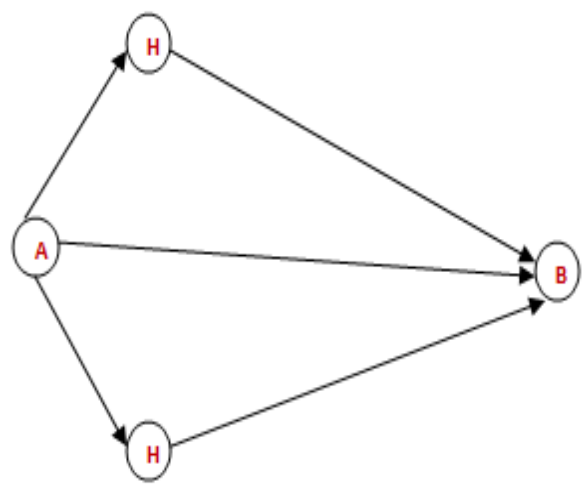

Fig-1: Extend the coverage using Cooperative Communication

\section{CURRENT SYSTEM}

The existing study relates to cooperative communication in the form of power saving and not that of prolong coverage.

Current research on topology control with cooperative communication recognizes the network connectivity for minimizing the transmission power of each and every node but ignores the energy efficient routes in constructed topologies. This may lead to faulty paths and damage the overall network performance in cooperative ad-hoc networks.

\section{PROJECTED SYSTEM}

We have proposed efficiency in two stages: The first stage relates energy efficient topology control and selection of optimum relay node. The second stage highlights two topology control algorithms for creating cooperative efficient energy spanners. To make the proposed algorithms simple, energetic and dynamic we recognize only the one-hop neighbors as accomplished helper nodes for each and every node when cooperative communication is used. As such the primary cooperative communication graph consists of all direct connections and cooperative communication links with one hop helpers as a substitute to all possible direct links and cooperative communication links. Both proposed algorithms are greedy algorithms. The main difference between the two is the processing order of links. The first algorithm excludes links greedily from the original graph while the second algorithm links into the graph greedily. Both algorithms can assure the property of the cooperative energy spanner of the constructed graph.

\section{IMPLEMENTATION}

\subsection{Greedy Algorithm}

An algorithm is a mathematical procedure generally known to deal with optimization issues in a limited number of steps. Most result algorithms make best depend on a global outline of all existing and future capabilities pointing at meeting the single global maximum result. Greedy algorithms proposed to solve certain class of optimization problems. A greedy algorithm finds a solution by proceeding one step at a time through the feasible solutions by applying a heuristic technique. Greedy algorithm works in two stages. In each stage, a decision is taken that seems to be good, without realizing about the consequences and it always arrive the best immediate solution. Greedy algorithms are simple and direct. Greedy algorithms are easy to implement. They require much less computing resources. They are much faster to execute. The term heuristic is used for algorithms which find solutions amidst all feasible ones. A well-arranged heuristic algorithm can achieve quality (near optimal) results. A heuristic approach assists to solve the problem by the method of trial and error. Example: Dijkstra's Algorithm for finding the shortest path. 


\subsection{Greedy Arrangement for Eliminating}

\section{Connections}

Let $\mathbf{S}$ be an empty graph. Then we add every direct connections $\mathbf{u}_{\mathbf{x}} \mathbf{u}_{\mathbf{y}}$ into $\mathbf{S}$, if node $\mathbf{U}_{\mathbf{X}}$ can arrives at node $\mathbf{U}_{\mathbf{Y}}$ in the meantime it performs with $\mathbf{P}_{\mathbf{M A X}}$. After that, for every couple of nodes $\mathbf{u}_{\mathrm{x}}$ and $\mathbf{u}_{\mathbf{y}}$, we choose a set of relay nodes $\mathbf{R}_{\mathbf{x y}}$ for node $\mathbf{u}_{\mathbf{x}}$ from its one-hop neighbors $\mathbf{N}\left(\mathbf{u}_{\mathbf{x}}\right)$, like that the link weight $\mathrm{w}\left(\mathbf{u}_{\mathbf{x}}, \mathbf{u}_{\mathbf{y}}\right)$ of the well-known Cooperative Communication connection is reduced. Note that this relay node collection difficulty is demanding even under our assumption that the transmission powers of $\mathbf{U}_{\mathbf{X}}$ and its relay node set to manage Cooperative Communication connection are the same. When we try all series of the relay sets to find the best relay set which decreases the overall energy consumption of $\mathbf{u}_{\mathbf{x}}$ and its relays, the computational difficulty is increasing to the range of the one-hop neighborhood $\mathrm{N}\left(\mathbf{u}_{\mathbf{x}}\right)$. It is not practical to do so in case of a huge number of neighbors. Hence, it is to exactly use the greedy heuristic algorithm Greedy Relay Set Selection $\left(\mathbf{u}_{\mathbf{x}}, \mathbf{N}\left(\mathbf{u}_{\mathbf{x}}\right), \mathbf{u}_{\mathrm{y}}\right)$; to choose the relay set $\mathbf{R}_{\mathbf{x y}}$. Later, correlate $\mathrm{w}\left(\mathbf{u}_{\mathbf{x}} \mathbf{u}_{\mathbf{y}}\right)$ with $\mathrm{p}$ (PG $\left.\left(\mathbf{u}_{\mathbf{y}}, \mathbf{u}_{\mathbf{y}}\right)\right)$ which is the present shortest path from node $\mathbf{u}_{\mathbf{x}}$ to node $\mathbf{u}_{\mathbf{y}}$ in $\mathbf{S}$. If $\mathrm{w}\left(\mathbf{u}_{\mathbf{x}} \mathbf{u}_{\mathbf{y}}\right) \leq \mathrm{p}\left(\mathrm{PG}\left(\mathbf{u}_{\mathbf{y}}, \mathbf{u}_{\mathbf{y}}\right)\right)$ and add this Cooperative Communication connection $\mathbf{u}_{\mathbf{x}} \mathbf{u}_{\mathbf{y}}$ into $\mathbf{S}$. If there already lies a direct connection $\mathbf{u}_{\mathbf{x}} \mathbf{u}_{\mathbf{y}}$, eliminate it after the new Cooperative Communication connection $\mathbf{u}_{\mathbf{x}} \mathbf{u}_{\mathbf{y}}$ is added.

\subsection{Greedy Arrangement for Adding Connections}

The alternative topology control algorithm starts with a sparse topology graph which is actively connected under Cooperative Communication model. The output of the algorithm can be used as the initial topology. Then, progressively add the utmost energy-efficient link into graph. Here, the energyefficiency of a link is represented as the gain for reducing energy span consideration by adding this link. Our algorithm will discontinue until the established graph satisfies the energy span consideration requirement. The overall gain of a link uxuy is the addition of the enhancement of bridge factors of every couple of nodes in graph after adding this link in each step; we ungenerously add the link with the largest bridgefactor-gain into graph. If there is a connection, we use the link weight to break it by adding the link with the least weight. We repeat this operation until graph meets the bridge factor requirement.

\subsection{Maximum Relay Nodes Selection}

Once communication topology is generated maximum nodes can be chosen from this topology for useful communication. As stated in Cooperative Communication model if $\mathbf{A}$ carry packets to $\mathbf{B}$ which is not in transmission range of $A$ due to saving power fixed transmission range, it can boost its transmission extend with the cooperation of its relay nodes and transmit packets. In this example node A utilizes it's all 1- hop neighbors though only few nodes are sufficient for sending data till $\mathbf{B}$.

\section{FLOW DIAGRAM}

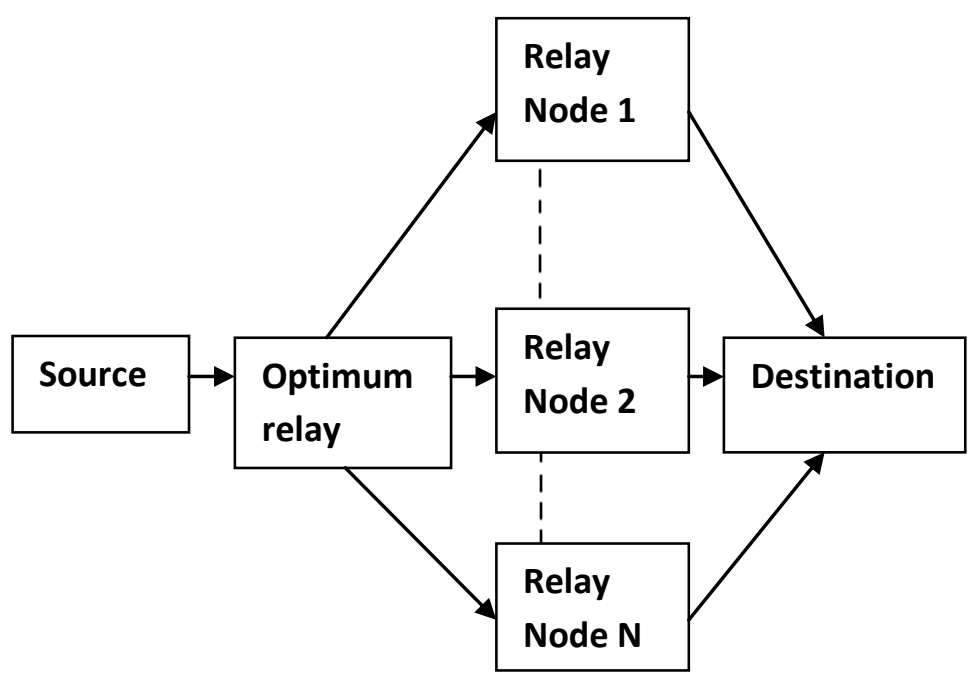

Fig-2: Flow Diagram

\section{SCREEN SHOTS}

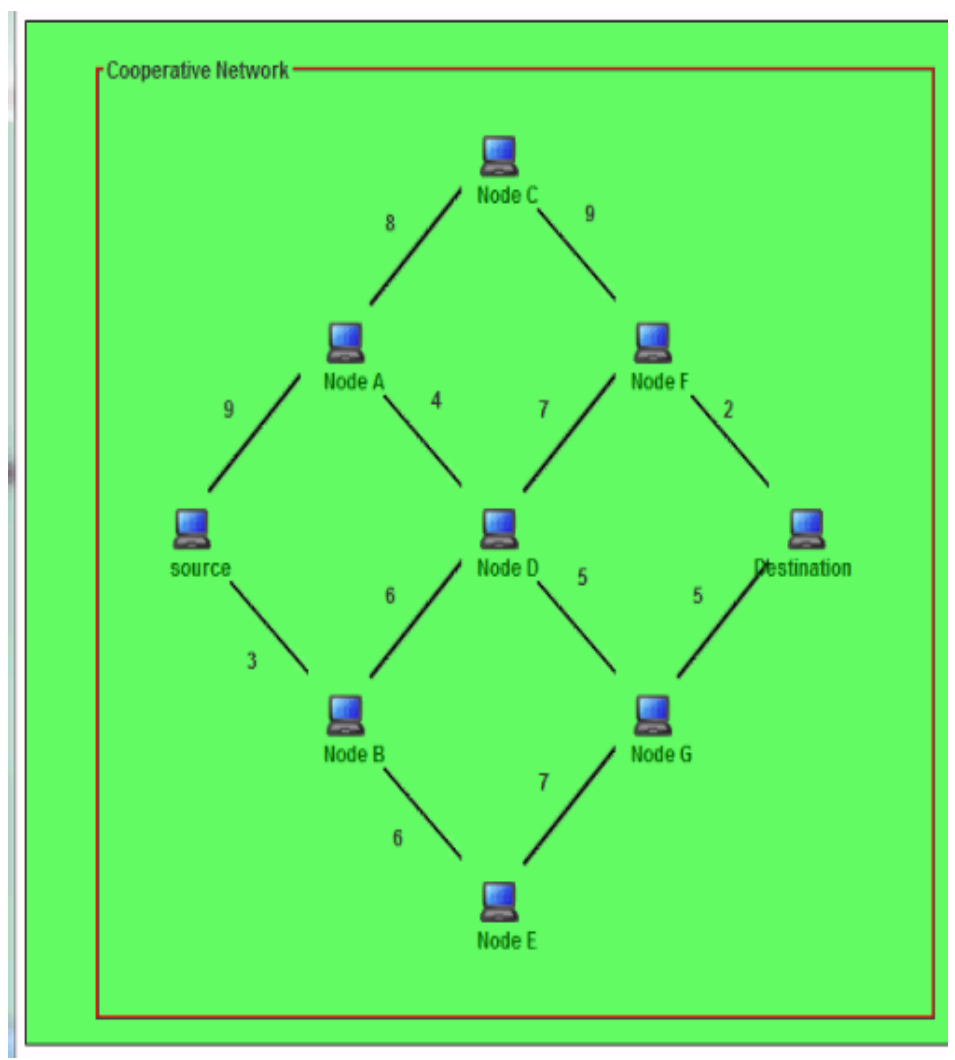

Fig-3: Cooperative Network 


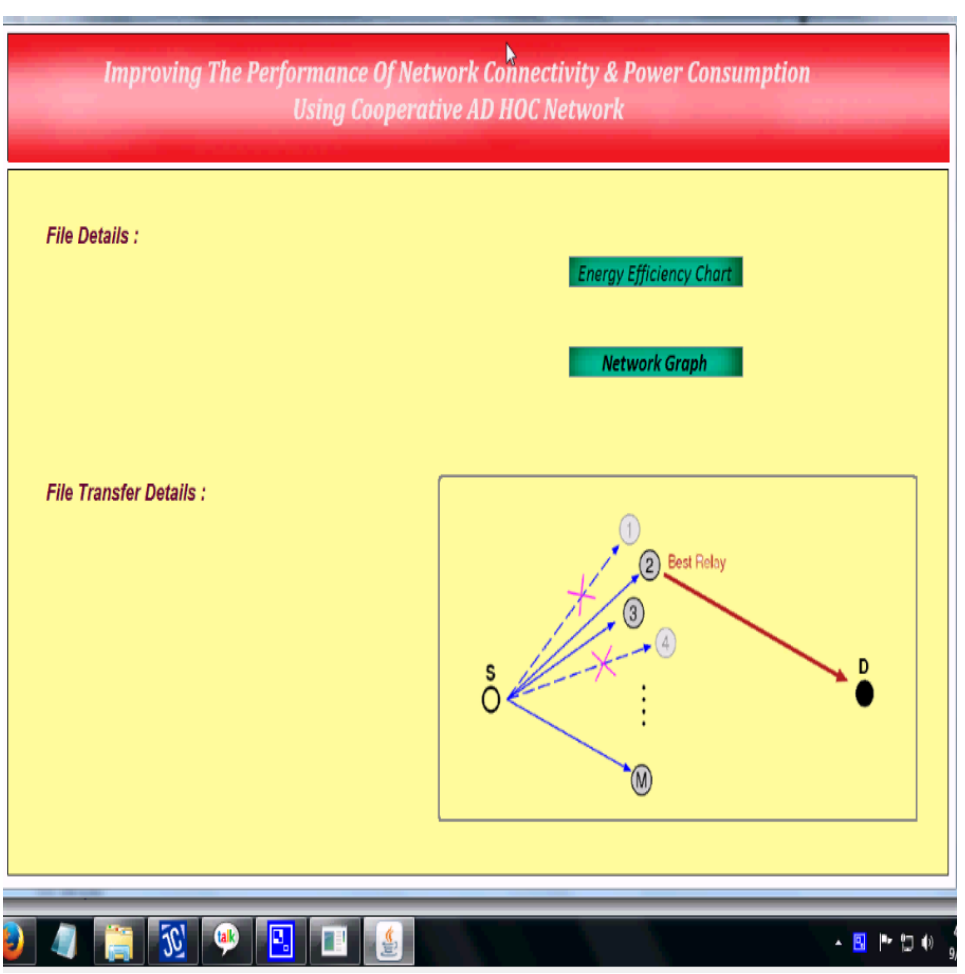

Fig-4: File details

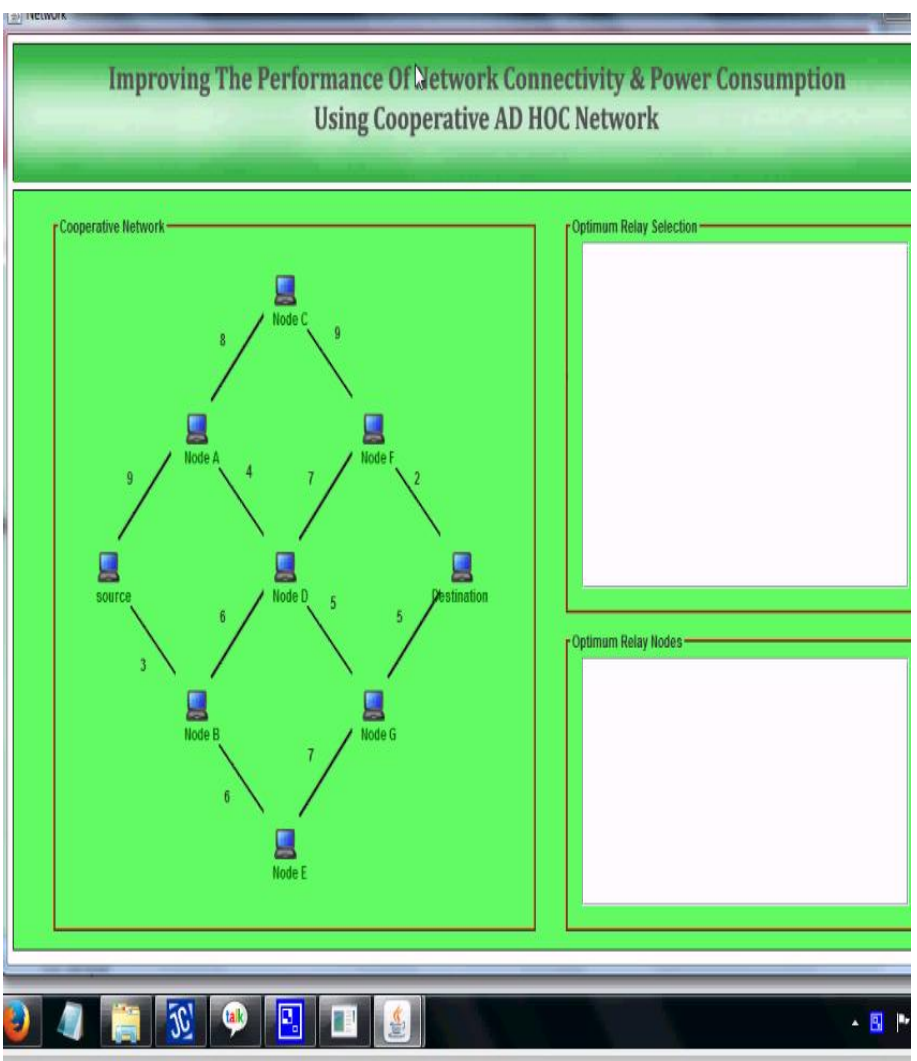

Fig-5: After Clicking the Network Graph Option

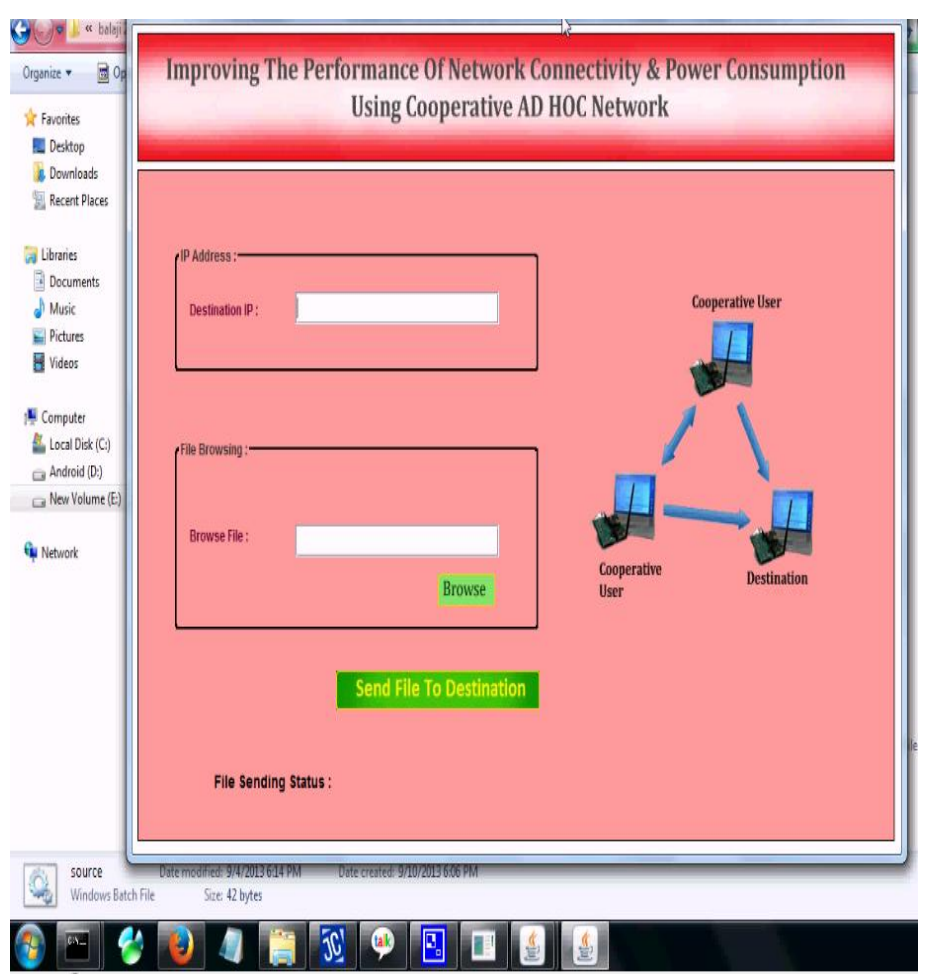

Fig-6: Ask for Destination IP Address

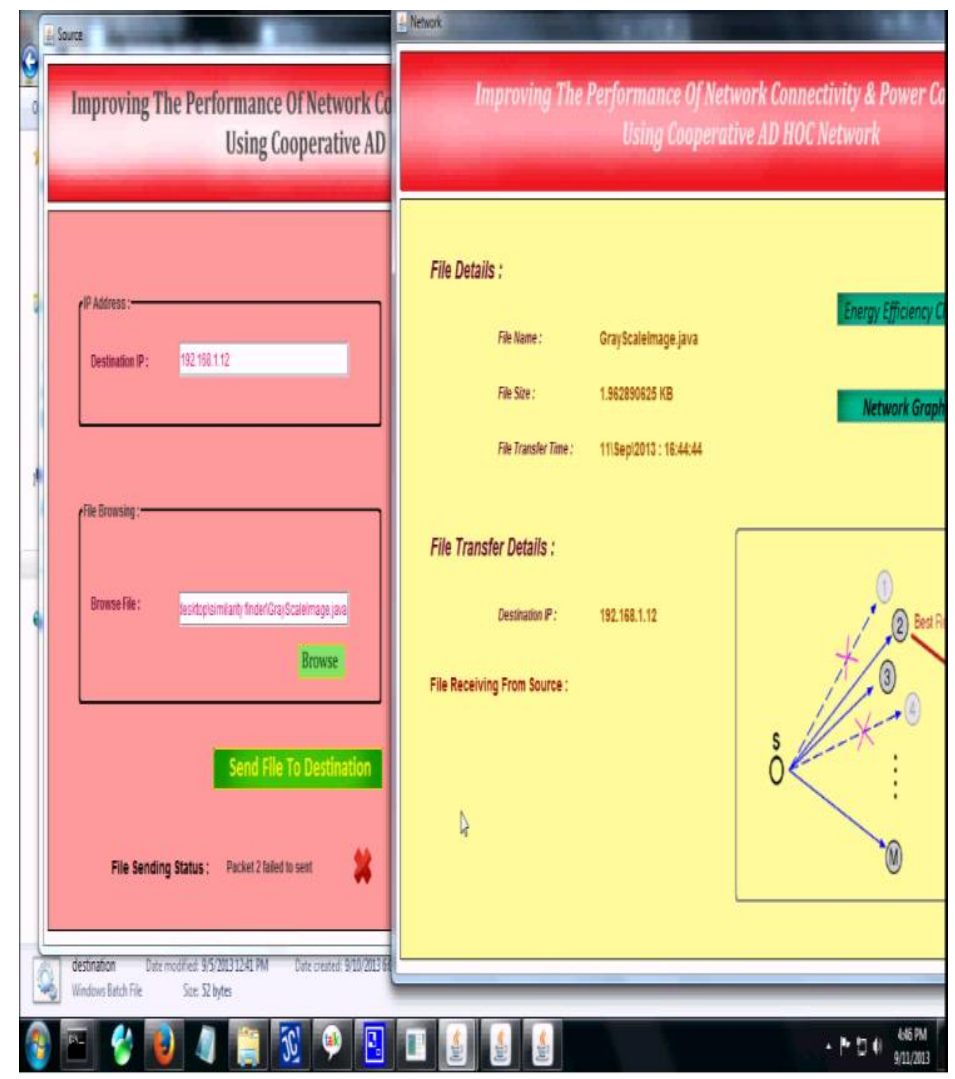

Fig-7: File Sending Status 


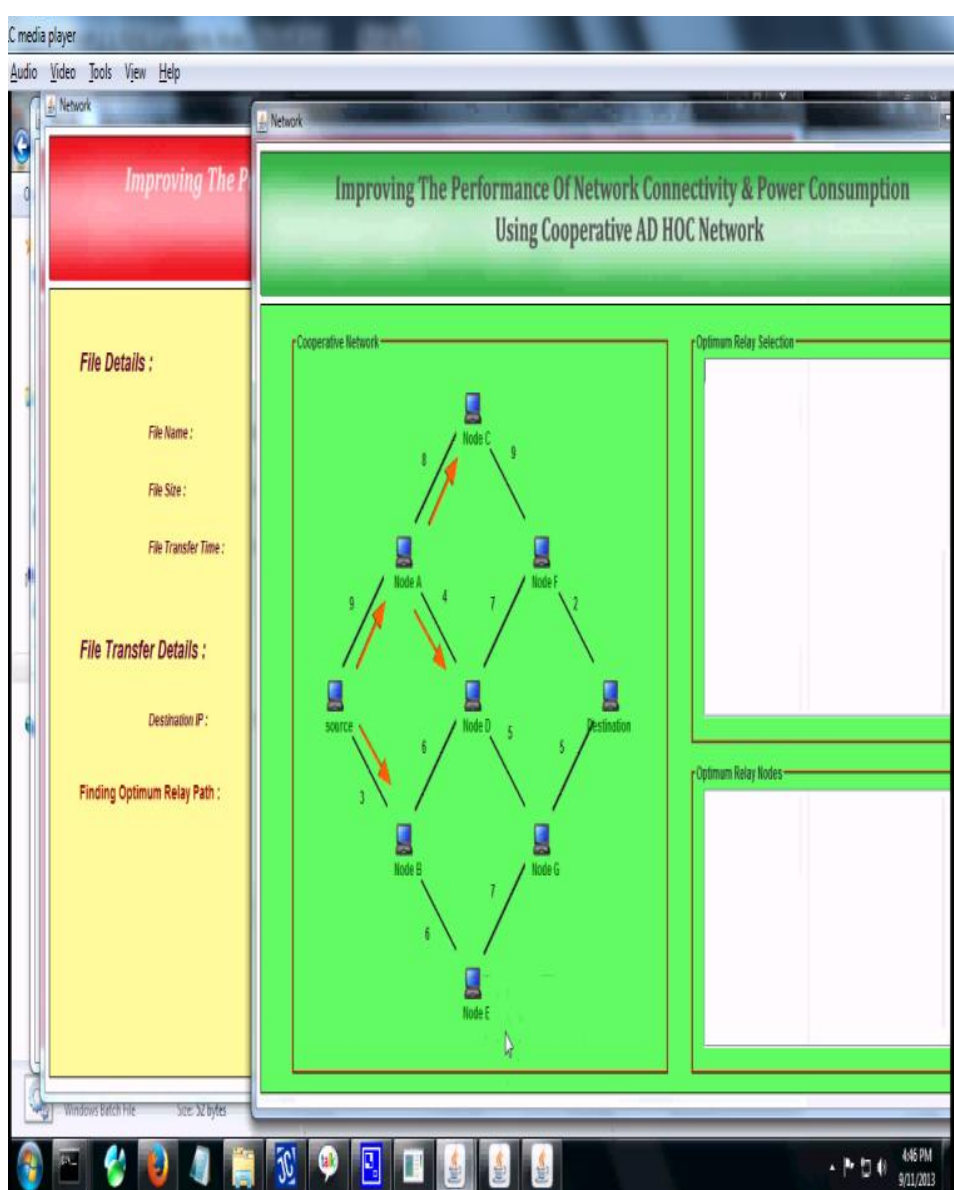

Fig-8: Flowing of data in the network

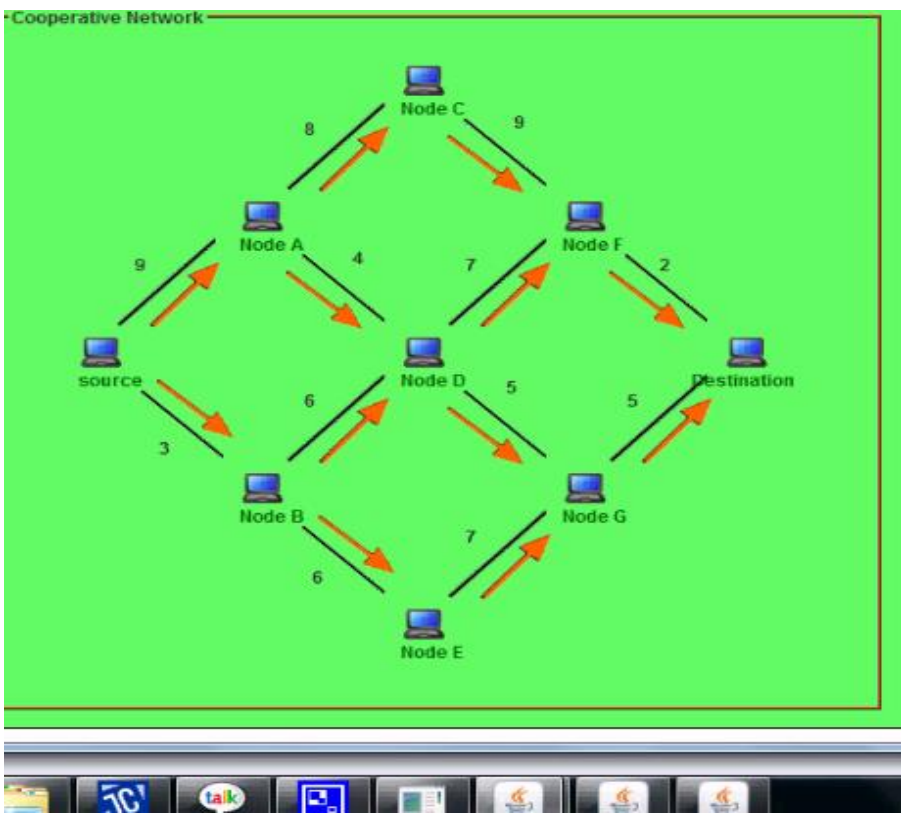

Fig-9:Data flow process

\section{CONCLUSIONS}

In wireless ad-hoc networks, Cooperative Communication plays a significant role. In this paper, we have proposed energy efficient topology control with Cooperative Communication and selection of optimum relay node. Cooperative Communication technique helps multiple nodes to transmit similar data at the same time. This increases the transmission coverage and also saves power.

\section{REFERENCES}

[1]. Ying Zhu, Minsu Huang, Siyuan Chen, and Yu Wang, "Energy-efficient topology control in cooperative ad hoc networks", vol. 23, no. 8, page 1480-1491, IEEE, 2012.

[2]. Quansheng Guan, Shengming Jiang, Victor C. M. Leung, "Topology control in mobile ad hoc networks with cooperative communication," IEEE 2012 Trans. on Wireless Communications, vol. 19.

[3]. Hiroki Nishiyama, Thuan Ngo, Nirwan Ansari and Nei Kato, "On minimizing the impact of mobility on topology control in mobile ad hoc networks," in IEEE Trans.on wireless communications, vol. 11, no. 3, March 2012.

[4]. A.K. Sadek, Z. Han, and K.J.R. Liu,"Distributed relayassignment protocols for coverage expansion in cooperative wireless networks," IEEE Trans. Mobile Computing, vol. 9, no. 4, pp. 505-515, Apr. 2010.

[5] Ying Zhu Minsu Huang Siyuan Chen Yu Wang, "Cooperative energy spanners: energy-efficient topology control in cooperative ad hoc networks", IEEE, 2010.

[6]. F. Hou, L.X. Cai, P.H. Ho, X. Shen, and J. Zhang, "A cooperative multicast scheduling scheme for multimedia services in ieee 802.16 networks," IEEE Trans. Wireless Comm., vol. 8, no. 3, pp. 1508-1519, Mar. 2009.

[7]. Ibrahim, Z.Han and K.Liu, "Distributed energy-efficient cooperative routing in wireless networks," IEEE Trans. Wireless Comm., vol. 7, no. 10, pp. 3930-3941, Oct. 2008.

[8]. J. Yu, H. Roh, W. Lee, S. Pack, and D.Z. Du, "Cooperative bridges: topology control in cooperative wireless ad hoc networks," in IEEE InfoCom, 2010.

[9]. L. Wang, B. Liu, D. Goeckel, D. Towsley, and C. Westphal, "Connectivity in cooperative wireless ad hoc networks," in ACM Mobihoc, 2008.

[10]. Lourthu Hepziba Mercy.M, Balamurugan.K, \& Vijayaraj.M, "Maximization of lifetime and reducing power consumption in wireless sensor network using protocol," in IJSCE vol.2, Jan 2013.

[11]. J. Bhuvaneswari, G.Rajkumar, C.Nishanthini, D.Sharmila , R.Sujitha, "Maximizing network capacity with topology control in cooperative communication," in IJET vol .5,no. 1, Feb-Mar 2013.

[12]. R. Bharathi, Saranya. A.V, "High throughput analysis using topological control \& authentication scheme in MANET," in IJETAE vol. 3, issue 3, March 2013.

[13] P. S. Karadge, Dr.S.V.Sankpal, "A performance comparison of energy efficient AODV protocols in mobile ad hoc networks," in IJARCCE vol. 2, issue 1, January 2013. 


\section{BIOGRAPHIES}

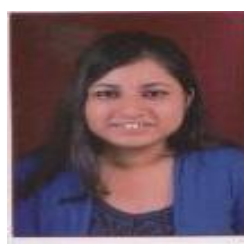

Miss Mohua Biswas, Assistant Professor in ETC department. Completed Bachelor degree from SVERI's C.O.E. Pandharpur. Now pursuing Master degree from SVERI's C.O.E. Pandharpur National Conference-01

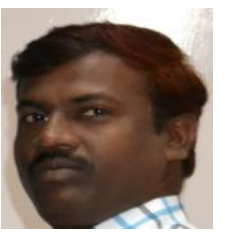

Name: Professor M.S.Mathpati, H.O.D of the ETC department having 13 years of Teaching Experience, National Conference 03 Subjects handled: Signal \& System, Linear Integrated Circuit \& Applications, RADAR Microwave Engineering, and Radio Frequency Microwave Circuit \& Design.

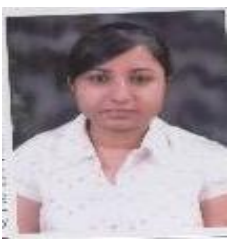

Name: Miss Papiya Biswas, Assistant Professor in ETC department. Completed Bachelor degree from SVERI's C.O.E. Pandharpur Now pursuing Master degree from SVERI's C.O.E. Pandharpur National Conference- 01 